\title{
2014 Gabriella E. Molnar, MD, Pediatric PM\&R Awards
}

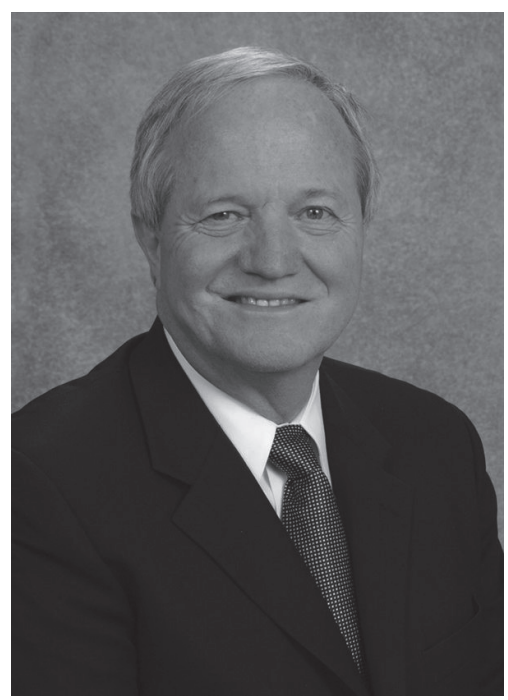

\section{Gabriella Molnar Pediatric PM\&R Lifetime Achievement Award}

Dr. Dennis Matthews, Fischahs Chair in Pediatric Rehabilitation and Medical Director of The Children's Hospital Rehabilitation Center at the University of Colorado Anschutz Medical Campus, was selected from among the nominees to receive this year's Gabriella Molnar, MD, Pediatric PM\&R Lifetime Achievement Award. His lifelong contributions to pediatric $P M \& R$ and $P M \& R$ education, the physiatric literature, and practice development have helped lay the foundation for our subspecialty and shaped the careers of countless pediatric PM\&R physicians. In addition, his involvement in positions of leadership within the AAPMR, AAP, ABPMR, and numerous other national medical organizations over the course of his career is unparalleled in our field. He received the award on Saturday, November 15 during the plenary session at the AAPM\&R Annual Assembly in San Diego.

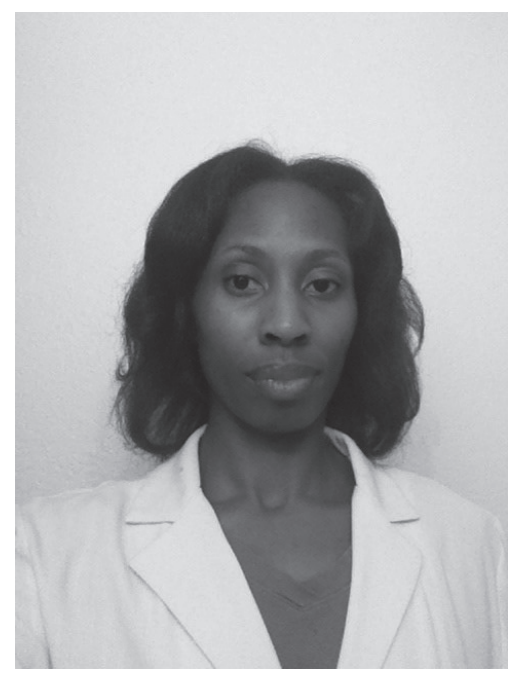

\section{Gabriella Molnar Pediatric PM\&R Research Grant}

Congratulations to Hadiya Green Guerrero, PT, DPT, from the Mayo Clinic for receiving the 2014 Gabriella Molnar Pediatric PM\&R Research Grant.

"Effectiveness and Efficacy of Kinesio Taping Method in Children Who Toe Walk"

The goals of her project are to: 1. Evaluate the effect of Kinesio Taping(KT) on ankle range of motion and ambulation in idiopathic toe walking. 2. Use Shear Wave Ultrasound Elastography(SWE) to quantify passive ankle muscle stiffness in children with ITW and 3. Compare SWE measurements before and after KT to changes in ambulation. 


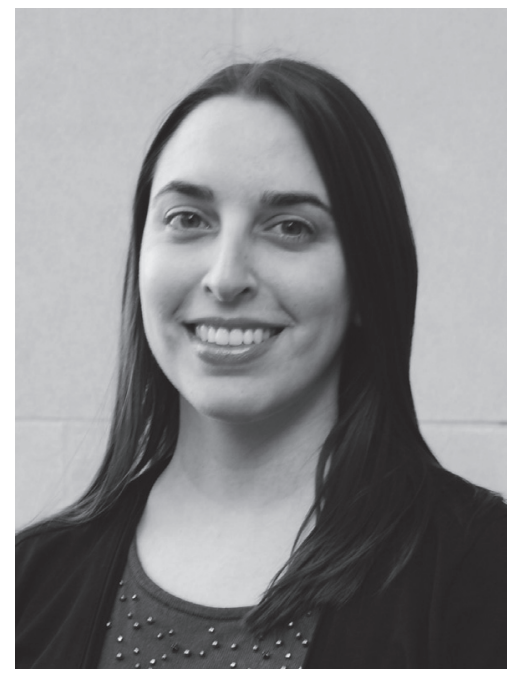

2014 Gabriella Molnar Transitional Care Research Grant

Congratulations to Kimberly Karrate, DO, from the University of North Carolina Center for Rehabilitation Care, for receiving the 2014 Gabriella E. MolnarSwafford Pediatric Transitional Care Research Grant. Titled Towards Transition and Self-management: Assessing the Needs of Individuals with Cerebral Palsy in North Carolina. Dr Karrate is a faculty member at the UNC School of Medicine.

The goals of her project are to: 1 . Investigate health transition and self-management needs, preferences, and experiences from the perspective of individuals with cerebral palsy and their families/caregivers. 2 . Develop a survey instrument to assess the health transition and self-management needs of individuals with cerebral palsy and their families/caregivers throughout North Carolina and 3. Conduct a survey to obtain information regarding the health transition and selfmanagement needs of individuals with cerebral palsy and their families/caregivers throughout North Carolina. 